\title{
The clinical use of right-sided double-lumen tubes
}

\author{
Peter Slinger, MD
}

Published online: 8 January 2010

(C) Canadian Anesthesiologists' Society 2010

Right-sided double-lumen tubes (DLTs) have a bad reputation. Many experts in the field of thoracic anesthesia regard them as being difficult to position, having a small margin of safety for correct placement, and being prone to intraoperative malpositioning. ${ }^{1}$ However, in a randomized prospective study, Campos et al. compared the use of right $v s$ left DLTs in two groups of 20 patients having left thoracotomies. ${ }^{2}$ In this small study, there were no significant differences between the two types of DLTs with respect to the relevant aspects of clinical performance, including the time for left lung collapse and the incidence of inadvertent right upper lobe collapse. There was a tendency for rightsided tubes to become malpositioned intraoperatively, but in all cases this difficulty was corrected easily by fibreoptic bronchoscopy. In a more recent retrospective report, the anesthetic records of 961 thoracic procedures were examined at one teaching institution where the usual practice was to use a DLT contralateral to the side of surgery, i.e., a rightsided tube for left-sided surgery. ${ }^{3}$ There were no differences in the incidences of hypoxemia, hypercarbia, or high airway pressures between patients managed with left or right DLTs. The current sales of DLTs in North America show a 10:1 preference for left- $v s$ right-sided tubes (personal communication with Covidien, Mansfield, MA, USA), suggesting that the majority of anesthesiologists tend to use left DLTs whenever possible. Given the evidence that right DLTs may function as well as left DLTs, and in view of the fact that it is necessary to use a right DLT in certain select cases, ${ }^{4}$

P. Slinger, MD $(\bowtie)$

Department of Anesthesia, Toronto General Hospital, University

of Toronto, Eaton North Wing, 3rd Floor, Room 441, 200

Elizabeth Street, Toronto, Ontario M5G 2C4, Canada

e-mail: peter.slinger@uhn.on.ca perhaps it is time to reconsider their use for routine airway management in thoracic surgery.

To appreciate our current clinical practice in thoracic anesthesia, it is useful to understand how we arrived at where we are today. Both the spectrum of patients and the equipment available for lung isolation have changed drastically from the origins of lung isolation in the 1930s. At that time, thoracic surgery was essentially for complications of infectious diseases, e.g., abscesses, bronchiectasis, hemoptysis, etc., which were often due to tuberculosis. The anesthesiologist's major priority was to protect the dependent lung from contamination with the infected secretions from the operative lung. The initial methods of lung isolation involved placement of a modified endotracheal tube as a single-lumen endobronchial tube in the mainstem bronchus of the non-operative lung. These tubes were placed by a rigid bronchoscope through the singlelumen tube and rigid bronchoscopy was a standard skill of the pioneers of thoracic anesthesia. ${ }^{5}$ Selective intubation of the contralateral lung became an established principle. In 1949, Carlens, a respirologist, introduced a red rubber leftsided DLT designed to be placed with topical anesthesia in awake patients with tuberculosis who were having splitlung broncho-spirometry as a part of their workup for possible lung resection. ${ }^{6}$ This DLT was quickly adopted by the thoracic surgeon, Bjork, who published a report with Carlens regarding the DLT's use in 500 pulmonary resections in $1952 .^{7}$ This landmark DLT had a carinal hook so it could be positioned solely with laryngoscopy and auscultation, eliminating the need for rigid bronchoscopy. It allowed simultaneous ventilation of the dependent lung and suctioning of the non-dependent lung, and it became the preferred device for lung isolation in the 1950's in preference to single-lumen tubes and bronchial blockers. Thus began the clinical preference for left DLTs. A right-sided version 
of the Carlens tube was introduced with a side-slot in the lateral wall of the distal bronchial lumen to ventilate the right upper lobe (the White tube), ${ }^{8}$ but it did not gain popularity because the bronchial cuff did not provide a reliable seal of the right mainstem bronchus. ${ }^{9}$

In the 1960's, Frank Robertshaw designed a series of red rubber DLTs without carinal hooks to facilitate intubation and with larger D-shaped lumens to decrease airflow resistance. ${ }^{10} \mathrm{He}$ designed both left- and right-sided DLTs, which allowed the pendulum of clinical practice to swing back to intubation of the contra-lateral bronchus. For his right DLT, he adapted the previous design of the GordonGreen right-sided single-lumen endobronchial tube with the ventilating side-slot contained within the bronchial cuff. All of the modern disposable DLTs are basically derivatives of Robertshaw's (unpatented) designs.

In the 1970's, disposable polyvinyl chloride (PVC) versions of DLTs were introduced. All of the left-sided commercial disposable DLTs conformed to Robertshaw's original design. However, for several engineering reasons, such as the decreased rigidity of the PVC DLTs, it was necessary for manufacturers to modify the right-sided Robertshaw DLT. The ventilating side-slot length and diameter were decreased to prevent the distal bronchial lumen from kinking (Figure 1). The aperture orifice of the side-slot was decreased by $>50 \%$. When these PVC right DLTs were placed blindly without fibreoptic surveillance, the reported incidence of right upper lobe obstruction was $89 \%$ vs $10 \%$ with red rubber Robertshaw right DLTs. ${ }^{11}$

During the 1980s, anesthesiologists debated the superiority of red-rubber vs PVC DLTs. ${ }^{12}$ However, by the 1990s, the hospital costs of re-sterilizing airway devices and the potential risks of contaminated reusable devices led to a dominance of PVC tubes in the developed world and the debate became moot. At approximately the same time, the cadaver studies by Benumof et al. highlighted the small room for error of right $v s$ left DLTs (1-4 mm $v s$ 16-19 mm, respectively), “...left-sided double-lumen tubes are much preferable to right-sided double-lumen tubes because they have a much greater positioning margin of safety...". ${ }^{13}$ At this time, since anesthesiologists in North America usually had access solely to PVC DLTs and since left-sided DLTs were clearly superior, the clinical pendulum swung back to where it currently rests, i.e., to the use of left-sided tubes whenever possible.

There is a spectrum of opinion on the use of right DLTs. The experts all agree that a right DLT is clearly the best option for lung isolation in some uncommon clinical situations. ${ }^{1,4}$ Such indications include a sleeve resection of the left mainstem bronchus (Figure 2), which may be performed for a lobar carcinoma that extends to the bronchial carina or a left mainstem endobronchial tumour, and in situations where the left mainstem bronchus is compressed by

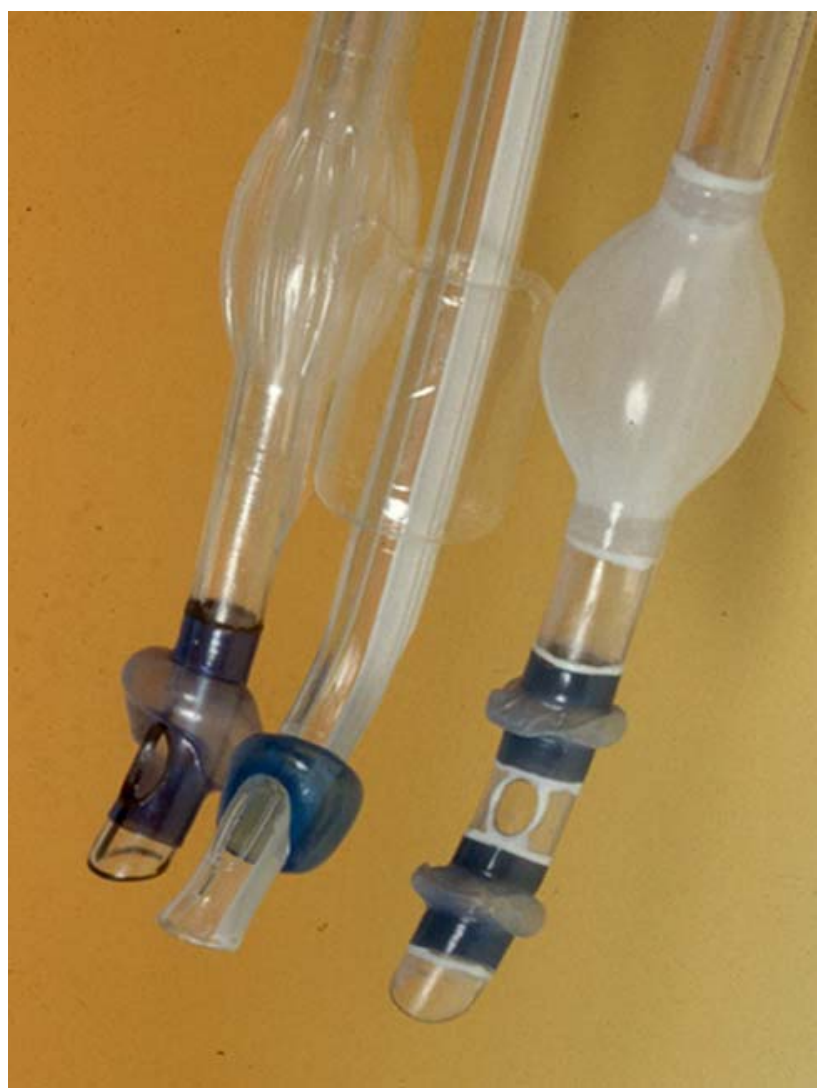

Fig. 1 A photograph of three commercial designs of right-sided double-lumen tubes: (left to right) Mallinckrodt (Mallinckrodt Canada Inc., Pointe Claire, QC, Canada), Rusch (Rusch Canada Inc., Oakville, ON, Canada), and Sheridan (Hudson RCI, Temecula CA, USA). All tubes incorporate a ventilating side-slot in the distal bronchial lumen to ventilate the right upper lobe. The side slots are smaller than those in the original Robertshaw design of the right double-lumen tube

a mediastinal mass or thoracic aortic aneurysm. The experts are diametrically opposed on the issue of using right-sided tubes for all left-sided thoracotomies. There is a grey area in-between, which is the use of right DLTs for left pneumonectomies, where a right DLT may offer some small clinical advantages by avoiding the need for repositioning when the left mainstem bronchus is clamped. Patients with a tracheal distortion, such as a "saber-sheath" trachea, present another situation wherein a right DLT may offer a benefit over a left DLT for a left thoracotomy. Some patients with severe chronic obstructive pulmonary disease develop a side-to-side compression of the intra-thoracic trachea. In the lateral position, the tracheal wall can partially obstruct the bevelled tracheal lumen of a DLT. ${ }^{14}$ Usually, this situation is just an inconvenience if the patient is being ventilated via the bronchial lumen during one-lung anesthesia, but it can be a serious clinical problem if the one-lung ventilation is via the tracheal lumen.

There is also a spectrum of opinion among thoracic surgeons on the use of right DLTs. Some surgeons who 


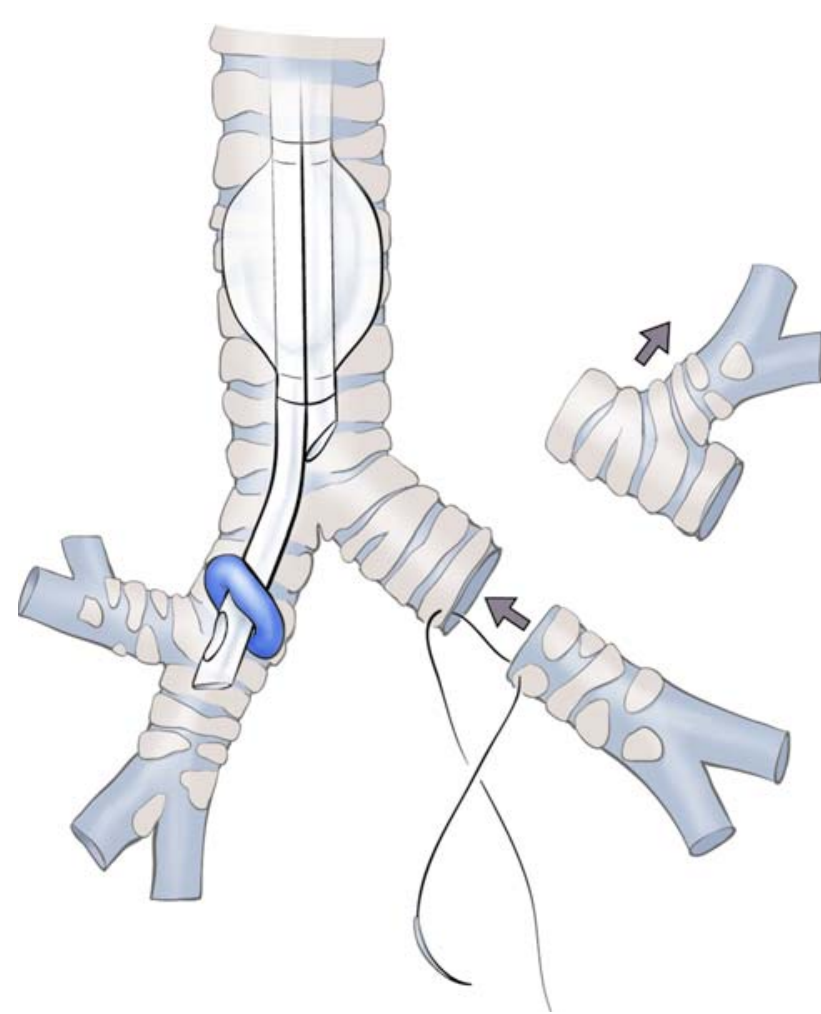

Fig. 2 Diagram of a left upper lobe sleeve resection. Indications for this type of resection would include a lobar carcinoma that extends into the mainstem bronchus. For surgery involving the left mainstem bronchus, a right double-lumen tube (DLT), as seen in the diagram, is the best option for airway management. A left DLT or bronchial blocker would interfere with surgery

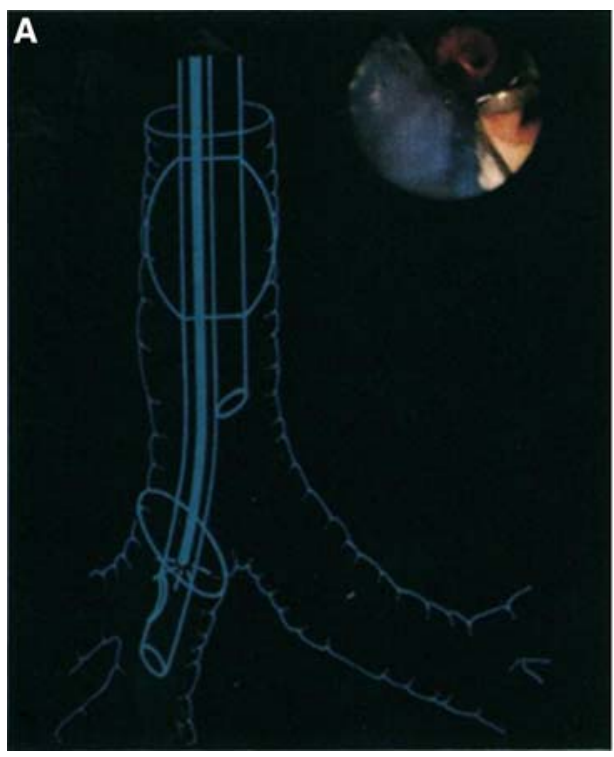

Fig. 3 Positioning of a right double-lumen tube (DLT). A: Diagram of a right DLT in the right mainstem bronchus. In the upper right corner is a photograph taken with a bronchoscope in the distal bronchial lumen. B: A diagram of the photograph in A. The right DLT is being rotated so that the partially obstructed right upper lobe bronchus is becoming visible in the side-slot of the bronchial lumen have had bad experiences with right DLTs are extremely negative. To date, these occurrences seem largely due to past use of right DLTs without appropriate fibreoptic positioning. In contrast, some surgeons actively encourage the use of right DLTs because they want their surgical residents to become familiar with them.

Several thoracic anesthesia centres in Canada (Quebec City and Vancouver) have attempted to improve the design of right DLTs with modified larger bronchial sideslots. ${ }^{15,16}$ Unfortunately, this effort has largely been met with inertia from the commercial tube manufacturers. Presumably, the right DLT market is too small to justify the expense of having a major modification approved by the governmental regulating agencies. Reusable and disposable right DLTs that conform to Robertshaw's original design are still produced (P3 Medical, Bristol, UK); however, these are not readily available in North America. Cost may be one factor that discourages clinicians from using right DLTs, as they tend to cost more per device than left DLTs. ${ }^{1}$

Like any device used for lung isolation, the fundamental "ABC"s apply. A is for anatomy. It is vital that anesthesiologists know the bronchial anatomy in order to position a right DLT so that the right upper lobe is not obstructed. However, many practicing anesthesiologists have a poor knowledge of fibreoptic bronchoscopic anatomy, since this anatomy was not routinely taught to anesthesia residents until recently. The results of one study in a North American teaching centre revealed a $38 \%$ incidence of staff anesthesiologists causing

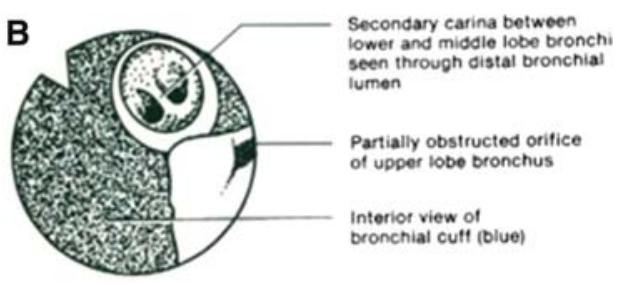

of the DLT. Note that the right upper lobe orifice is located on the lateral wall of the mainstem bronchus directly in line with the lateral end of the secondary carina between the middle and lower lobes. (Reproduced with permission from: Slinger P. Fiberoptic positioning of double-lumen tubes. J Cardiothorac Anesth 1989; 3: 486-96.) 
major initial malpositioning of DLTs and blockers, and the authors found these miscalculations were largely due to anesthesiologists' inadequate knowledge of bronchial anatomy. ${ }^{17}$ To help practitioners learn this anatomy, our institution has produced a free online bronchoscopy simulator on our website, www.thoracicanesthesia.com.

$\mathrm{B}$ is for bronchoscope. When positioning a right DLT, it is important to use a fibreoptic bronchoscope at all times (Figure 3). In all likelihood, fibreoptic bronchoscopy has been the major factor enabling clinicians to return to the routine use of right DLTs for left-sided surgery despite the acknowledged problems with their current design. The advent of video-bronchoscopy has been another factor which has encouraged the use of right DLTs. With a videobronchoscope, the anesthesiologist, resident, and surgeon can all watch as a tube is correctly positioned.

$\mathrm{C}$ is for chest $\mathrm{X}$-ray and computed tomography scan. It is important for the anesthesiologist (him/herself) to look at the chest imaging prior to induction of anesthesia to confirm that there is normal tracheo-bronchial anatomy. Anatomical abnormalities, such as a tracheal take-off of the right upper lobe bronchus (occurs in an estimated $0.4 \%$ of patients) ${ }^{18}$ or compression of the right mainstem bronchus that would contraindicate the use of a right DLT, can be appreciated from the imaging in advance.

In summary, there is evidence that right-sided tubes can function well for left-sided surgery. ${ }^{2,3}$ An important caveat is that the authors who have reported these results work in high-volume thoracic centres and are experienced thoracic anesthesiologists. Since all residents may have to use a right DLT, possibly in a trying situation, anesthesiologists in teaching centres should endeavour to see that residents gain experience positioning right DLTs whenever a suitable clinical indication for lung isolation arises.

\section{L'utilisation clinique des sondes à double lumière droites}

Les sondes à double lumière (SDL) droites ont mauvaise réputation. Plusieurs experts en anesthésie thoracique pensent qu'elles sont difficiles à positionner, parce qu'elles n'ont qu'une marge de sécurité restreinte pour être bien placées et sont sujettes aux mauvais positionnements pendant l'opération. ${ }^{1}$ Cependant, dans une étude randomisée prospective, Campos et coll. ont comparé l'utilisation des SDL droites et gauches dans deux groupes de 20 patients subissant des thoracotomies sur le côté gauche. ${ }^{2}$ Dans cette petite étude, il n'y a pas eu de différence significative entre les deux types de SDL en ce qui concerne les aspects pertinents de la performance clinique, notamment le temps jusqu'au collapsus du poumon gauche et l'incidence de collapsus involontaire du lobe supérieur droit. Les sondes droites ont eu tendance à se déplacer pendant l'opération, mais cette complication a été aisément corrigée dans tous les cas à l'aide d'une bronchoscopie à fibres optiques. Dans un compte-rendu rétrospectif plus récent, les dossiers anesthésiques de 961 chirurgies thoraciques ont été passés en revue dans un hôpital universitaire où la pratique courante consistait à utiliser une SDL du côté opposé au site de la chirurgie, soit une sonde droite pour une chirurgie sur le côté gauche. ${ }^{3}$ Cette étude n' a pas observé de différence en matière d'incidences d'hypoxémie, d'hypercarbie ou de pression élevée des voies aériennes entre les patients chez qui on avait placé une SDL gauche ou droite. Les ventes actuelles de SDL en Amérique du Nord montrent une préférence de 10 contre 1 pour les sondes gauches (communication personnelle avec Covidien, Mansfield, MA, USA), ce qui laisse supposer que la majorité des anesthésiologistes privilégieront l'utilisation de SDL gauches chaque fois que cela est possible. Au vu des données probantes soutenant que les SDL droites sont tout aussi performantes que les SDL gauches, et étant donné qu'il est nécessaire d'utiliser une SDL droite dans certains cas précis, ${ }^{4}$ il est peut-être temps de repenser leur utilisation lors de la prise en charge de routine des voies aériennes dans les cas de chirurgie thoracique.

Pour bien saisir en quoi consiste notre pratique clinique actuelle en anesthésie thoracique, il importe de comprendre le cheminement que nous avons fait pour y parvenir. L'éventail des patients et du matériel à notre disposition pour isoler les poumons ont radicalement changé depuis les débuts de la thoracoscopie dans les années 1930. À cette époque, la chirurgie thoracique était réalisée quasi exclusivement pour traiter des complications liées à des maladies infectieuses, comme par exemple les abcès, la bronchiectasie, ou encore l'hémoptysie-certaines des complications fréquentes de la tuberculose. La priorité numéro un de l'anesthésiologiste était alors de protéger le poumon du côté en décubitus d'une contamination causée par les sécrétions infectées du poumon opéré. Les premières techniques d'isolement du poumon impliquaient le placement d'une sonde endotrachéale modifiée en tant que sonde endobronchique à lumière unique dans la bronche souche du poumon non opéré. Ces sondes étaient enfilées dans la sonde à lumière unique grâce à un bronchoscope rigide, la bronchoscopie rigide étant une compétence standard de ces pionniers de l'anesthésie thoracique. ${ }^{5}$ L'intubation sélective du poumon opposé est devenue un principe bien établi. En 1949 Carlens, un pneumologue, a introduit une SDL gauche en caoutchouc rouge, laquelle avait été conçue pour être placée sous anesthésie topique chez des patients éveillés souffrant de tuberculose et subissant une bronchospirométrie séparée dans le cadre de leur préparation à une possible résection pulmonaire. ${ }^{6}$ Cette SDL a été rapidement adoptée par 
Bjork, le chirurgien thoracique qui en 1952 a publié avec Carlens un compte-rendu concernant l'utilisation de cette SDL dans 500 cas de résection pulmonaire. ${ }^{7}$ Cette SDL a marqué l'histoire : elle disposait d'un crochet caréné de manière à pouvoir être positionnée à l'aide de laryngoscopie et d'auscultation seulement, éliminant ainsi la nécessité d'une bronchoscopie rigide. Cette sonde permettait une ventilation du poumon dépendant et la succion simultanée du poumon non dépendant; elle est devenue l'appareil de choix pour la thoracoscopie dans les années 50, avant l'apparition des sondes à lumière unique et des bloqueurs bronchiques. C'est ainsi que la préférence pour les SDL gauches a commencé dans la pratique clinique. Une version droite de la sonde de Carlens a été introduite. Elle comprenait une fente de côté dans la paroi latérale de la lumière bronchique distale qui permettait de ventiler le lobe supérieur droit (la sonde de White) ${ }^{8}$ mais elle n'a pas conquis le marché clinique parce que le ballonnet bronchique n'isolait pas la bronche souche droite de façon fiable. ${ }^{9}$

Dans les années 60, Frank Robertshaw a conçu une série de SDL de caoutchouc rouge sans crochet caréné afin de faciliter l'intubation, lesquelles disposaient également de lumières plus larges en forme de $\mathrm{D}$ afin de réduire la résistance à l'écoulement d'air. ${ }^{10}$ Il a conçu des SDL gauches et droites, ce qui a eu pour conséquence que la tendance de la clinique pratique s'est à nouveau tournée vers une intubation de la bronche opposée au côté de l'opération. Pour concevoir sa SDL droite, Robertshaw a adapté le modèle précédent de sonde endobronchique à lumière unique droite de Gordon-Green en lui ajoutant la fente latérale de ventilation contenue dans le ballonnet bronchique. En fait, toutes les SDL modernes jetables sont dérivées des modèles (non brevetés) de Robertshaw.

Dans les années 70, on a introduit des versions jetables des SDL en PVC (polychlorure de vinyle). Toutes les SDL gauches jetables commercialisées suivaient le modèle original de Robertshaw. Cependant, pour plusieurs raisons techniques, notamment en raison de la rigidité moindre des SDL en PVC, il a fallu que les fabricants modifient la SDL droite de Robertshaw. La longueur et le diamètre de la fente de ventilation latérale ont été réduits afin d'éviter que la lumière bronchique distale ne se plie (Figure 1). L'orifice d'ouverture de la fente latérale a été réduit de $>50 \%$. Lors du positionnement en aveugle de ces SDL droites en PVC sans vérification par fibroscopie, l'incidence rapportée d'obstruction du lobe supérieur droit a atteint $89 \%$, contre $10 \%$ avec des SDL droites en caoutchouc rouge de Robertshaw. ${ }^{11}$

Dans les années 80, les anesthésiologistes ont étudié la supériorité présumée des SDL en caoutchouc rouge par rapport à celles en PVC. ${ }^{12}$ Toutefois, dans les années 90 , les coûts hospitaliers liés à la re-stérilisation des appareils

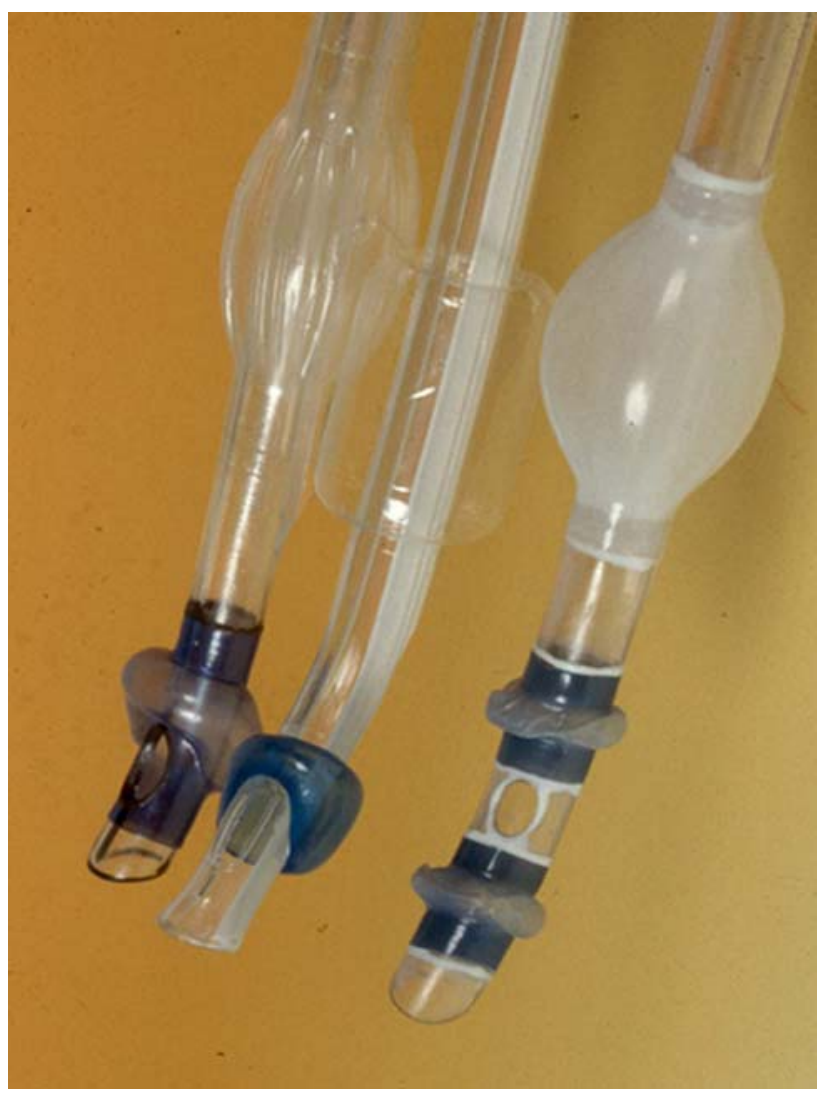

Fig. 1 Photographie de trois modèles commerciaux de sondes à double lumière droites: (de gauche à droite) Mallinckrodt (Mallinckrodt Canada Inc., Pointe Claire, QC, Canada), Rusch (Rusch Canada Inc., Oakville, ON, Canada), et Sheridan (Hudson RCI, Temecula CA, États-Unis). Toutes les sondes comprennent une fente latérale de ventilation dans la lumière bronchique distale de façon à ventiler le lobe supérieur droit. Les fentes latérales sont plus petites que celles du modèle original de sonde à double lumière droite de Robertshaw

pour les voies aériennes et les risques potentiels de contamination des appareils réutilisables ont fait pencher la balance en faveur des sondes en PVC dans les pays industrialisés; le débat a donc perdu sa raison d'être. Plus ou moins au même moment, les études sur cadavres de Benumof et coll. soulignaient la petite marge d'erreur laissée par les SDL droites comparativement aux gauches (1-4 mm vs 16-19 mm, respectivement) : «les sondes à double lumière gauches sont nettement préférables aux sondes à double lumière droites parce qu'elles offrent une marge de sécurité de positionnement bien plus étendue... ». ${ }^{13}$ À cette époque, étant donné que les anesthésiologistes avaient en général uniquement accès à des SDL en PVC en Amérique du Nord et étant donné que les SDL gauches étaient clairement supérieures, la tendance clinique a basculé à nouveau vers la position où elle se situe actuellement, soit vers l'utilisation de sondes gauches à chaque fois que cela est possible. 


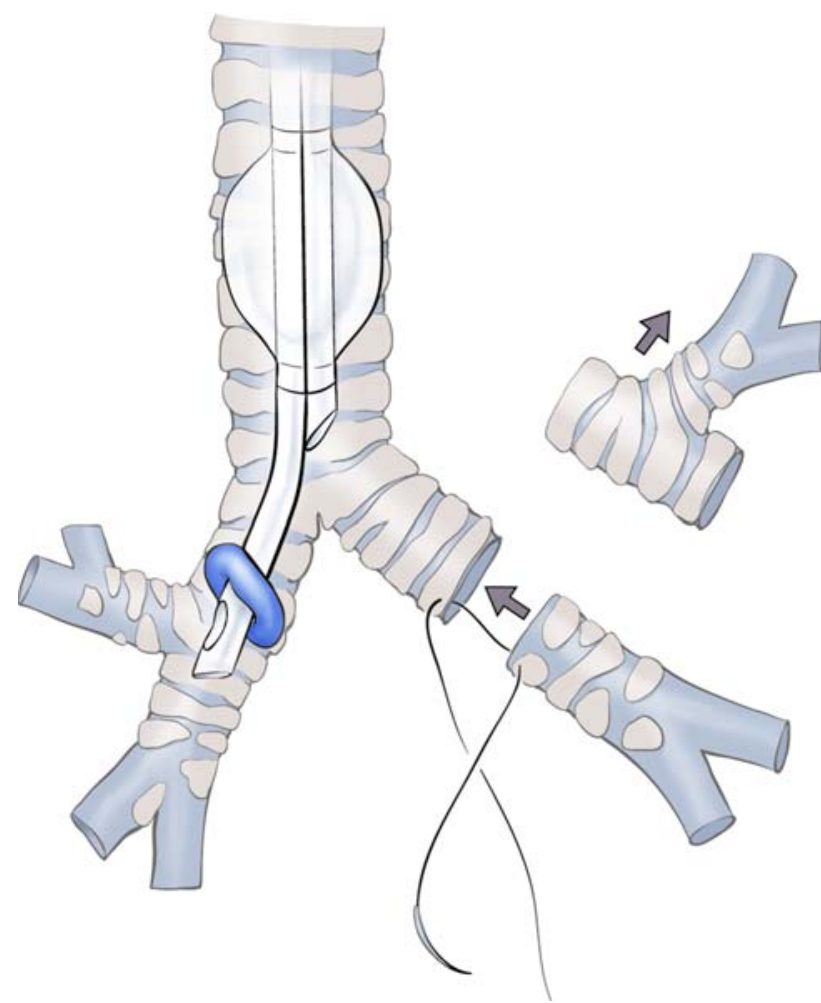

Fig. 2 Schéma d'une résection « en manche » du lobe supérieur gauche. Parmi les indications de ce type de résection figurent un carcinome lobaire s'étendant dans la bronche souche. Dans les cas de chirurgie impliquant la bronche souche gauche, une sonde à double lumière (SDL) droite, telle que présentée dans le schéma, constitue la meilleure option pour la prise en charge des voies aériennes. Une SDL gauche ou un bloqueur bronchique gêneraient la chirurgie

Toutes sortes d'opinions existent quant à l'utilisation des SDL droites. Les experts sont tous d'avis qu'une SDL droite constitue clairement la meilleure alternative pour isoler le poumon dans certaines situations cliniques rares. ${ }^{1,4}$ Parmi ces indications, citons les cas de résection «en manche» de la bronche souche gauche (Figure 2), laquelle peut être réalisée pour traiter un carcinome lobaire qui s'étend à la carène bronchique d'une tumeur endobronchique de la souche gauche, ainsi que dans les situations où la bronche souche gauche est comprimée par une masse médiastinale ou un anévrisme de l'aorte thoracique. Par contre, les opinions des experts sont diamétralement opposées au sujet de l'utilisation de sondes droites pour toutes les thoracotomies gauches. Au milieu se trouve une zone grise en ce qui concerne l'utilisation de SDL droites pour les pneumonectomies gauches, situation dans laquelle une SDL droite pourrait présenter certains petits avantages cliniques en éliminant le besoin de repositionnement lors du clampage de la bronche souche gauche. Les patients présentant une distorsion trachéale comme par exemple une trachée en «fourreau d'épée » sont un autre cas dans lequel une SDL droite pourrait être avantageuse comparée à une SDL gauche pour une thoracotomie gauche. Certains patients souffrant de maladie pulmonaire obstructive grave manifestent une compression latérolatérale de la trachée thoracique. En position latérale, la paroi trachéale peut obstruer partiellement la lumière trachéale biseautée d'une SDL. ${ }^{14}$ En règle générale, ce genre de cas n'est qu'un désagrément si le patient est ventilé par lumière bronchique pendant une anesthésie unipulmonaire, mais cela peut constituer un problème clinique grave si la ventilation unipulmonaire se fait par la lumière trachéale.

Les opinions sur l'utilisation des SDL droites divergent également parmi les chirurgiens thoraciques. Certains chirurgiens ayant eu de mauvaises expériences avec les SDL droites sont extrêmement négatifs. À ce jour, ces événements semblent causés principalement par l'utilisation passée de SDL droites sans repérage adéquat par fibroscopie. En revanche, d'autres chirurgiens sont de fervents défenseurs des SDL droites parce qu'ils souhaitent que leurs résidents en chirurgie sachent les utiliser.

Plusieurs centres d'anesthésie thoracique au Canada (Québec et Vancouver) ont tenté d'améliorer la conception des SDL droites en en élargissant les fentes latérales bronchiques. ${ }^{15,16}$ Malheureusement, ces efforts n'ont eu que très peu d'échos chez les fabricants de sondes commerciales. Vraisemblablement, le marché des SDL droites est trop restreint pour justifier les dépenses liées à une modification majeure approuvée par les organismes gouvernementaux de réglementation. On produit encore des SDL droites, réutilisables ou jetables, sur la base du modèle original de Robertshaw (P3 Medical, Bristol, R.-U.); cependant, elles ne sont pas disponibles en Amérique du Nord. Le coût pourrait être l'un des facteurs décourageant les cliniciens de l'utilisation de SDL droites, étant donné qu'elles coûtent en général environ $50 \%$ de plus que les sondes gauches. ${ }^{1}$

Comme tout appareil utilisé pour isoler les poumons, les « $\mathrm{ABC}$ » de base s'appliquent. A comme anatomie. Il est indispensable que les anesthésiologistes connaissent l'anatomie des bronches s'ils veulent positionner une SDL droite de façon à ne pas obstruer le lobe supérieur droit. Pourtant, plusieurs praticiens n'ont qu'une connaissance limitée de l'anatomie bronchoscopique par fibroscopie; en effet, jusqu'à récemment cette anatomie ne faisait pas partie du cursus normal des résidents en anesthésie. Les résultats d'une étude menée dans un centre universitaire nord-américain ont révélé que dans $38 \%$ des cas, les anesthésiologistes membres du personnel étaient à l'origine d'un mauvais positionnement majeur des SDL et des bloqueurs. Les auteurs ont découvert que ces erreurs étaient principalement dues au manque de connaissances en anatomie bronchique des anesthésiologistes. ${ }^{17}$ Afin d'aider les praticiens à se familiariser avec cette anatomie, notre 


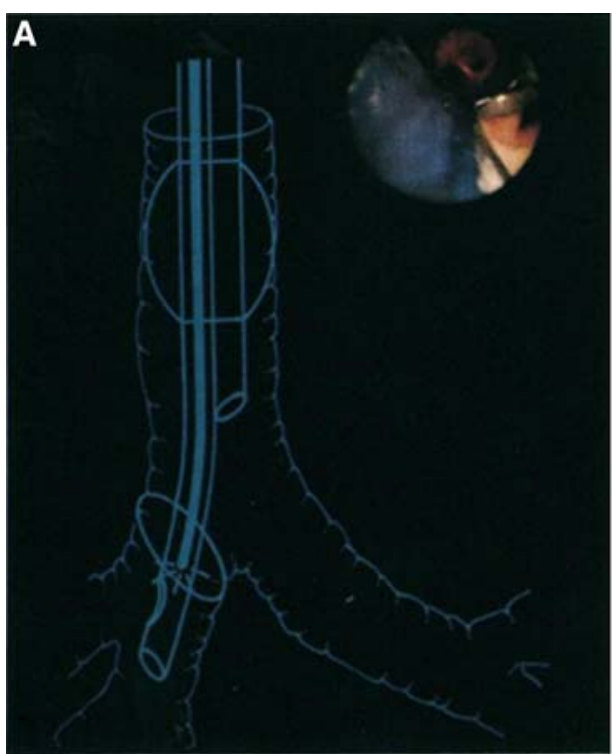

Fig. 3 Positionnement d'une sonde à double lumière (SDL) droite. A : Schéma d'une SDL droite dans la bronche souche droite. Dans le coin supérieur droite se trouve une photographie prise à l'aide d'un bronchoscope dans la lumière bronchique distale. B : Schéma de la photographie présentée en A. La SDL droite est tournée de manière à ce que la bronche du lobe supérieur droit, partiellement obstruée, devienne visible dans la fente latérale de la lumière bronchique de la

institution a créé un simulateur de bronchoscopie gratuit en ligne, lequel est disponible sur notre site Internet au www.thoracicanesthesia.com.

B comme bronchoscope. Lors du positionnement d'une SDL droite, il est important d'utiliser en tous temps un bronchoscope à fibres optiques (Figure 3). La bronchoscopie à fibres optiques a selon toute vraisemblance été le facteur principal du retour des cliniciens à une utilisation de routine des SDL droites pour les chirurgies sur le côté gauche et ce, malgré les problèmes que l'on connaît à leur conception actuelle. La venue de la vidéo-bronchoscopie est un autre facteur ayant encouragé l'utilisation de SDL droites. Avec un bronchoscope vidéo, l'anesthésiologiste, le résident et le chirurgien peuvent tous observer pendant que la sonde est bien positionnée.

Enfin, il y a la radiographie des poumons et la tomodensitométrie. Il est important que l'anesthésiologiste regarde lui-même les radiographies des poumons avant l'induction de l'anesthésie. Ainsi, il pourra confirmer que l'anatomie trachéo-bronchique est normale. En effet, les anomalies anatomiques comme l'embranchement trachéal de la bronche lobaire supérieure droite (incidence estimée à $0,4 \%$ des patients) ${ }^{18}$ ou la compression de la bronche souche droite qui contre-indiqueraient l'utilisation d'une SDL droite peuvent être évaluées à l'avance grâce à l'imagerie.

En bref, plusieurs données probantes soutiennent que les sondes droites peuvent être utiles lors d'une chirurgie sur le

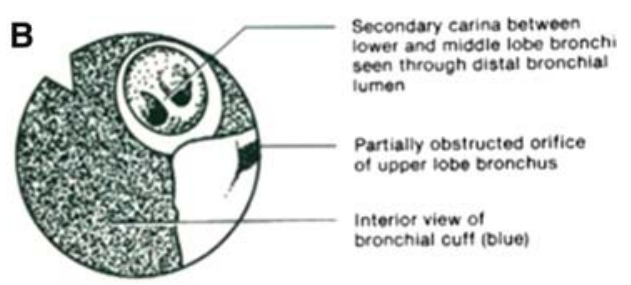

SDL. Il est intéressant de noter que l'orifice du lobe supérieur droit est situé sur la paroi latérale de la bronche souche, directement aligné avec l'extrémité latérale de la carène secondaire, entre les lobes médian et inférieur. (Reproduit avec la permission de : Slinger P. Fiberoptic positioning of double-lumen tubes. J Cardiothorac Anesth 1989; 3: 486-96)

côté gauche. ${ }^{2,3}$ Mais il faut tenir compte du fait que les auteurs ayant rapporté ces résultats travaillent dans des centres où l'on fait beaucoup de chirurgies thoraciques et sont par là même des anesthésiologistes expérimentés. Étant donné que tous les résidents seront peut-être appelés à utiliser une SDL droite un jour, et possiblement dans une situation d'urgence, il est du devoir des anesthésiologistes travaillant dans des centres universitaires de s'assurer que les résidents acquièrent suffisamment d'expérience dans le positionnement des SDL droites à chaque fois qu'une indication clinique adaptée à l'isolement du poumon survient.

Acknowledgements The author sincerely thanks Dr. George Lee, Consultant Anaesthetist, Wythenshaw Hospital, Manchester, England and Dr. Ian Conacher, retired Consultant Anaesthetist, Freeman Hospital, Newcastle, England for the background information they contributed to this article.

Competing interests None declared.

\section{References}

1. Cohen E. Con: right-sided double-lumen endotracheal tubes should not be routinely used in thoracic surgery. J Cardiothorac Vasc Anesth 2002; 16: 249-52.

2. Campos JH, Massa FC, Kernstine KH. The incidence of right upper-lobe collapse when comparing a right-sided double-lumen tube versus a modified left double-lumen tube for left-sided thoracic surgery. Anesth Analg 2000; 90: 535-40. 
3. Ehrenfeld JM, Walsh JL, Sandberg WS. Right- and left-sided Mallinckrodt double-lumen tubes have identical clinical performance. Anesth Analg 2008; 106: 1847-52.

4. Campos JH, Gomez MN. Pro: right-sided double-lumen endotracheal tubes should be routinely used in thoracic surgery. J Cardiothorac Vasc Anesth 2002; 16: 246-8.

5. Mushin WM, Rendall-Baker L. The Origins of Thoracic Anesthesia. Park Ridge, IL: Wood Library Museum of Anesthesiology; 1991: 128-32

6. Carlens E. A new flexible double-lumen catheter for bronchospirometry. J Thorac Surg 1949; 18: 742-6.

7. Bjork VO, Carlens E, Friberg O. Endobronchial anesthesia. Anesthesiology 1953; 14: 60-72.

8. White GM. A new double lumen tube. Br J Anaesth 1960; 32: 232-4.

9. Pappin JC. The current practice of endobronchial intubation. Anaesthesia 1979; 34: 57-64.

10. Robertshaw FL. Low resistance double-lumen endobronchial tubes. Br J Anaesth 1962; 34: 576-9.

11. McKenna MJ, Wilson RS, Botelho RJ. Right upper lobe obstruction with right-sided double-lumen tubes: a comparison of two tube types. J Cardiothorac Anesth 1988; 2: 734-40.
12. Burton NA, Watson DC, Brodsky JB, Mark JB. Advantages of a new polyvinyl chloride double-lumen tube in thoracic surgery. Ann Thorac Surg 1983; 36: 78-84.

13. Benumof JL, Partridge BL, Salvatierra C, Keating J. Margin of safety in positioning modern double-lumen endotracheal tubes. Anesthesiology 1987; 67: 729-38.

14. Bayes J, Slater EM, Hedberg PS, Lawson D. Obstruction of a double-lumen endotracheal tube by a saber-sheath trachea. Anesth Analg 1994; 79: 186-8.

15. Bussieres JS, Lacasse Y, Cote D, et al. Modified right-sided Broncho-Cath double-lumen tube improves endobronchial positioning: a randomized study. Can J Anesth 2007; 54: 276-82.

16. Lohser J, Umedaly HS, Fitzmaurice BG. Do-it-yourself modified right-sided Broncho-Cath double lumen tube. Can J Anesth 2007; 54: 675.

17. Campos JH, Hallam EA, Van Natta T, Kernstine KH. Devices for lung isolation used by anesthesiologists with limited thoracic experience: comparison of double-lumen endotracheal tube, Univent torque control blocker, and Arndt wire-guided endobronchial blocker. Anesthesiology 2006; 104: 261-6.

18. Brodsky $J B$, Mark $J B$. Bilateral upper lobe obstruction from a single double-lumen tube. Anesthesiology 1991; 74: 1163-4. 